\title{
Efficacy of Saline Injection Therapy for Atrophic Acne Scars
}

\author{
Sidra Khan, Rabia Ghafoor and Sana Kaleem \\ Department of Dermatology, Jinnah Postgraduate Medical Centre, Karachi, Pakistan
}

\begin{abstract}
Objective: To determine the efficacy of saline injection in post-acne atrophic scars on face.

Study Design: Non-randomized clinical trial.

Place and Duration of Study: Department of Dermatology, Jinnah Postgraduate Medical Centre, Karachi, from January to July 2019.

Methodology: All patients with mild, moderate and severe post-acne atrophic scars, above the age of 15 years without any comorbid conditions were included in the study. Intra-dermal isotonic saline solution was administered under local anesthesia, into the scars weekly for 12 weeks. The results were assessed subjectively and objectively by photographs, Sharquie scoring system and Dermatology Life Quality Index (DLQI) at baseline and at the end of treatment. SPSS 23 was used for analyzing data.

Results: Out of 49 patients, 41 (83.7\%) were females and 8 (16.3\%) were males. The mean age was $24.69 \pm 4.35$ years. According to Sharquie scoring system of grading scars at baseline, $3(6.1 \%), 34(69.4 \%)$ and $12(24.5 \%)$ patients had mild, moderate and severe scarring respectively while at the end of treatment, $35(71.4 \%), 12(24.5 \%)$ and $2(4.1 \%)$ patients had mild, moderate and severe scarring respectively $(p=0.001)$. Regarding degree of satisfaction of patients, $6(12.2 \%), 27(55.1 \%), 12(24.5 \%)$ and $4(8.2 \%)$ patients were mildly, moderately, greatly and completely satisfied respectively. The mean DLQI was $12.14 \pm 3.29$ and $5.86 \pm 2.26$ at baseline and at the end of treatment respectively $(p=0.001)$.

Conclusion: The response of saline injection was significant in all types of post-acne atrophic scars as assessed with Sharquie score and DLQI, but more significant in mild and moderate scars, without any significant side effects.
\end{abstract}

Key Words: Acne, scars, saline solution.

How to cite this article: Khan S, Ghafoor R, Kaleem S. Efficacy of Saline Injection Therapy for Atrophic Acne Scars. J Coll Physicians Surg Pak 2020; 30(04):359-363. DOI: https://doi.org/10.29271/jcpsp.2020.04.359.

\section{INTRODUCTION}

Acne is an inflammatory process, which is multi-factorial involving increased sebum production at puberty due to androgenic activity, alteration in the quality of sebum lipid production, colonisation of Propioni bacterium acnes and occlusion of follicular duct by keratin plug. ${ }^{1}$ It involves areas of body with high concentrations of pilo-sebaceous glands particularly face, back and chest. It is characterised by polymorphic lesions like papules, pustules, nodules, cysts, and open and close comedones. Healing leaves erythema, pigmentation and either atrophic or hypertrophic scars depending on the net loss or gain of collagen. ${ }^{2}$ Atrophic scars are more common than hypertrophic scars and keloids.

Atrophic scars are depressed scars, in which all layers of skin are intact and appear as invagination on surface. They result after resolution of inflammatory phase of acne and healing of an injury below the normal level of skin surface. The severity of scarring depends on the degree of inflammatory process of the damaged tissue and time lapsed from the onset of inflammatory process. ${ }^{3}$ Severe scarring results in psychological distress particularly in adolescents. ${ }^{4}$

Correspondence to: Dr. Sidra Khan, Department of Dermatology, Jinnah Postgraduate Medical Centre, Karachi, Pakistan

E-mail:dr.sidrakhan786@gmail.com

Received: January 07, 2020; Revised: March 12, 2020;

Accepted: March 31, 2020

DOI: https://doi.org/10.29271/jcpsp.2020.04.359
Intradermal saline injection for post-acne atrophic scars is one of the most cost effective and safest methods as there are no side effects reported so far when compared with other treatment modalities for resurfacing post-acne atrophic scars like microneedling, subcision, lasers, chemical peels, dermabrasion and dermal fillers. These are expensive and require expertise. ${ }^{5}$ They have side effects and not always fulfill patients' expectations. In this study, the efficacy of intradermal and subdermal saline injection for post-acne atrophic scars was assessed with Sharquie scoring system for grading scars and Dermatology Life Quality Index (DLQI). No such study is carried out before, in our setup. The objective of this study was to determine the efficacy of saline injection in post-acne atrophic scars on face.

\section{METHODOLOGY}

This prospective non-randomized clinical study was conducted in the Department of Dermatology of Jinnah Postgraduate Medical Centre, Karachi from January to July 2019 after receiving approval from the institutional ethical and research committee of hospital and registeration with the international RCT registry (ClinicalTrials.gov Identifier NCT04197245). The sample size was calculated through NCSS software. Forty-nine non-admitted Dermatology patients were selected by purposive non-probability sampling. Those patients aged between 15-35 years of either gender with post-acne atrophic scars were included in the study. Patients with hypertension, cardiovascular disorder, pregnancy, lactation and hypersensitivity to lidocaine were excluded. 
Table I: Sharquie scoring for grading scars.

\begin{tabular}{|c|c|c|c|c|}
\hline Score parameter & 1 & 2 & 3 & 4 \\
\hline No. of scars & $<10$ & $11-20$ & $21-30$ & $>30$ \\
\hline Total area of scars & $<1 / 4$ & $1 / 4-1 / 2$ & $1 / 2-3 / 4$ & $>3 / 4$ \\
\hline Type of scars & Flat & Depressed & Hypertrophic & Keloid \\
\hline Colour of scars & Skin coloured & $\begin{array}{l}\text { Erythematous / } \\
\text { hypopigmented }\end{array}$ & Hyperpigmented & Bluish / greyish \\
\hline Effect on psyche & None / mild discomfort & Mild dysmorphophobia & Moderate dysmorphophobia & $\begin{array}{l}\text { Severe dysmorphophobia / } \\
\text { social withdraw }\end{array}$ \\
\hline
\end{tabular}

Table II: Descriptive statistics of scoring system.

\begin{tabular}{|l|l|l|}
\hline Parameters & Mean \pm SD & Paired samples test $($ sig) \\
\cline { 1 - 2 } Sharquie score before treatment & $12.73 \pm 2.33$ & \multirow{2}{*}{$\mathrm{t}=16.481, \mathrm{p}=0.001$} \\
\cline { 1 - 2 } Sharquie score after treatment & $8.67 \pm 2.30$ & \\
\cline { 1 - 2 } DLQI score before treatment & $12.14 \pm 3.29$ & \multirow{2}{*}{$\mathrm{t}=18.833, \mathrm{p}=0.001$} \\
\cline { 1 - 2 } DLQI score after treatment & $5.86 \pm 2.26$ & \\
\hline
\end{tabular}

\section{DLOISCORE}

1. Over the last week, how itchy, sore, painful or stinging has you r skin been? verymuch •A lot •A little *Not at all •Notrelevant

2. Over the last week, how embarrassed or self-conscious have you been because of your skin?

-verymuch •Alot •Alittle •Not at all •Notrelevant

3. Over the last week, how much has your skin interfered with you going shopping or looking after you rhome or garden?

verymuch •Alot •Alittle •Not at all •Notrelevant

4. Over the last week, how much has your skin influenced the dothes you wear? -verymuch •Alot •Alittle •Not at all •Notrelevant

5. Over the last week, how much has your skin affected any social or leisure activities?

-verymuch -A lot $\cdot$ A little Not at all -Notrelevant

6. Over the last week, how much has your skin made it difficult for you to do any sport?

-verymuch •Alot •Alittle •Not at all -Notrelevant

7. Over the last week, has your skin prevented you from working or stud ying? If "No", over the last week how much has your skin been a problem at work or studying?

-verymuch •Alot •Alittle •Not at all •Notrelevant

8. Over the last week, how much has your skin created problems with your partner or any of your dose friends or relatives?

-verymuch •Alot $\cdot$ Alittle Not at all *Notrelevant

9. Over the last week, how much has your skin cau sed any sexual difficulties? -verymuch •Alot $\cdot$ Alittle $\quad$ Not at all $\quad$ Notrelevant

10. Over the last week, how much of a problem has the treatment for your skin been, for exam ple by making you r home messy, or by taking up time?

verymuch -A lot -A little *Not at all "Notrelevant

Figure 1: Dermatology Life Quality Index Questionnaires.

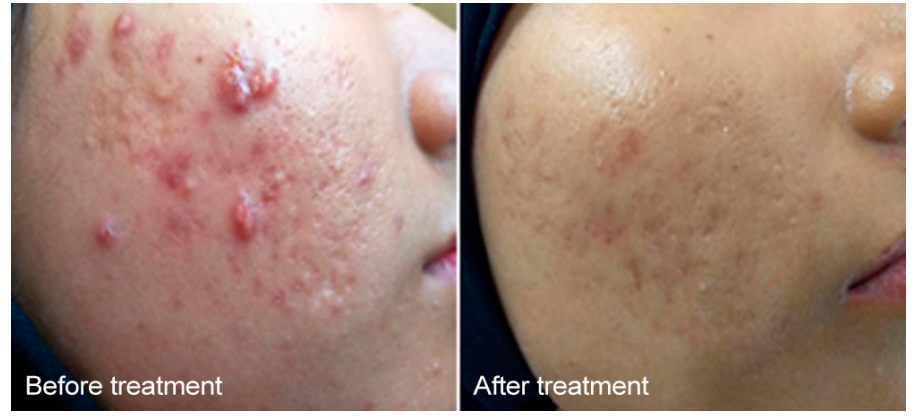

Figure 2; Appearance at baseline and after treatment.
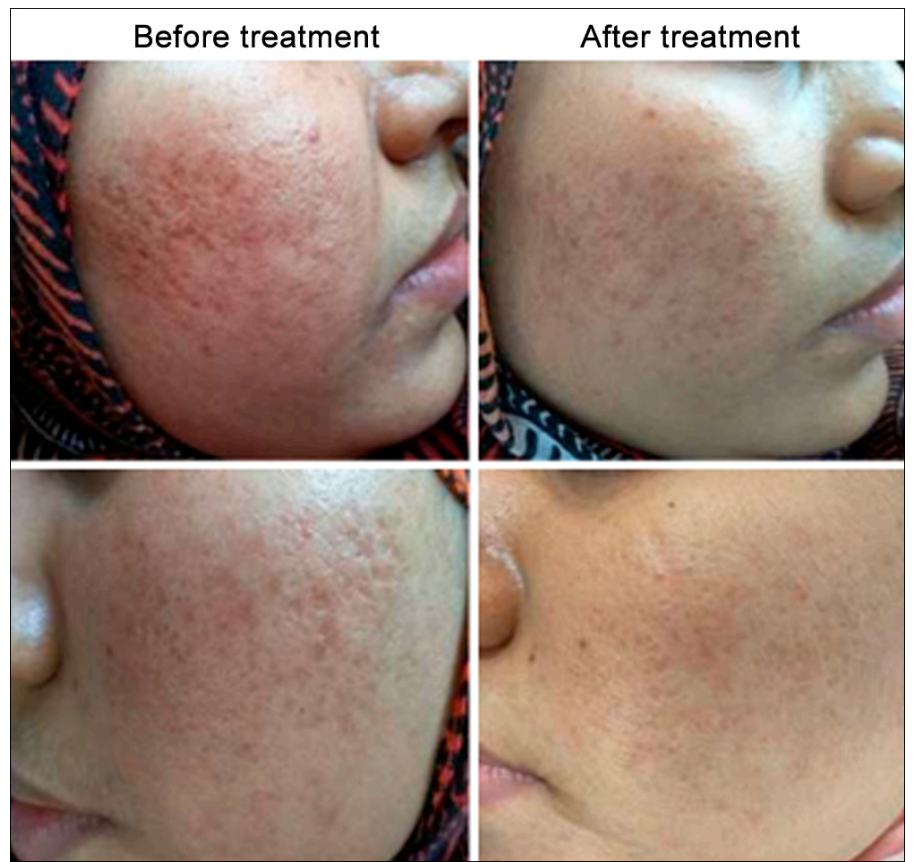

Figure 3: Appearance at baseline and after treatment.

The procedure was explained to each patient and written informed consent was taken. Before starting treatment, the photographs were taken for each patient with their permission and Sharquie scoring system for grading scars (flat, atrophic, hypertrophic or keloid) and Dermatology Life Quality Index (DLQI) were calculated.

During each session, after local sterilization and infiltration with lidocaine, $0.9 \%$ isotonic saline was injected intra- and sub-dermally into atrophic acne scars with 1cc syringe of 30 gauge needle, on both sides of the face; injected up to the point of scar elevation as well as spread of saline into the 
surrounding tissues. The volume of saline injected depended on the number, width and depth of individual scar. A total of 12 sessions were done weekly. The efficacy of treatment was assessed objectively by comparing photographs and Sharquie scoring system while subjectively by the degree of patients' satisfaction and DLQI at the start and at the end of treatment. The Sharquie scoring system for grading scars was calculated through different scoring parameters, illustrated in Table I. ${ }^{6}$ After adding all parameters of scoring system, the final scores were interpreted as mild, moderate and severe if they have scores in between 5-9, 10-14 and 15-20 respectively. The final scores were compared with the initial ones, at the end of treatment.

The Dermatology Life Quality Index (DLQI) was calculated. This index signifies the effect of disease, effect of treatment on daily activities and the impact on social behavior of the patient. DLQI scores were interpreted as 0-1 (No effect), 2-5 (small effect), 6-10 (moderate effect), 11-20 (very large effect) and 21-30 (extremely large effect). At the end of treatment results were compared with that of the baseline to assess the improvement in everyday activities and social behavior of the patients. ${ }^{7}$

The qualitative variables were expressed as frequency along with percentages of patients according to severity of scars and the effect on quality of life and subjected to analysis through chi-square test. The quantitative variables were expressed as mean along with standard deviation (SD) of the scores and analysed with paired t test. The statistical analysis was done through IBM SPSS statistics software version 23. The level of significance was set at $p<0.05$. The side effects were also assessed regarding erythema, itching, pain and abnormal pigmentation.

\section{RESULTS}

Forty nine patients were enrolled with clinical diagnosis of post-acne atrophic scars, 41 (83.7\%) were females and 8 $(16.3 \%)$ were males with age ranging from 18 to 35 years (mean $=24.69 \pm 4.35$ years). Mean ages in females and males were $25 \pm 4.08$ and $23 \pm 5.52$ years respectively. Mean duration of post-acne atrophic scars was $4.95 \pm 3.96$ years.

According to Sharquie scoring system for grading atrophic scars, at baseline 3 (6.1\%) patients had mild, 34 (69.4\%) patients had moderate and $12(24.5 \%)$ patients had severe scarring. At the end of treatment, 35 (71.4\%) patients had mild, $12(24.5 \%)$ patients had moderate and $2(4.1 \%)$ patients had severe scarring (Chi-square $=18.722^{\mathrm{a}}, \mathrm{p}=0.001$ ).

Regarding patients' degree of satisfaction, 6 (12.2\%) patients were mildly satisfied, $27(55.1 \%)$ patients were moderately satisfied, $12(24.5 \%)$ patients were greatly satisfied, and 4 $(8.2 \%)$ patients were completely satisfied with the treatment of saline injection.

The Dermatology Life Quality Index (DLQI) scores at baseline showed 15 (30.6\%) patients had moderate effect, 33 (67.3\%) patients had very large effect and 1 (2\%) patient had extremely large effect while after treatment, it showed 27
(55.1\%) patients had small effect, $20(40.8 \%)$ patients had moderate effect and 2 (4.1\%) patients had very large effect on their daily activities and social behaviour. Chi-square = $41.081^{a}, p=0.001$.

After treatment mean scores were significantly lower as illustrated in Table II. The mean difference was $4.061 \pm 1.725$ for Sharquie score and $6.286 \pm 2.336$ for DLQI; confidence level set at $95 \%$, with confidence interval of $3.566-4.557$ and 5.615 - 6.957 for Sharquie score and DLQI respectively.

During treatment, reduction in eruption of new acne lesions, healing of old lesions, improvement in post-acne pigmentation and skin tightening was also observed (Figures 1 and 2). No side effects were observed.

\section{DISCUSSION}

Scars are formed by replacement of normal tissue with fibrous tissue as a result of trauma, infection or inflammation. ${ }^{8}$ Acne is an inflammatory condition and degree of severity of scars depends on the depth and extent of the inflammatory process. Inflammation that remains confined to epidermis or superficial dermis, results in macules which may be erythematous, hypopigmented or hyperpigmented. When deep dermis is involved, it results in scar formation either atrophic or hypertrophic. ${ }^{9}$ Atrophic scars can be classified as Rolling, Icepick and Boxcar scars according to their depth of involvement of epidermis, dermis and subcutaneous tissue. ${ }^{10}$

This trial is carried out for the first time in Karachi. In this study, response of saline injection in all types of atrophic scars was significant, but it was more pronounced in mild to moderate scars. The probable mechanism of intra-dermal saline injection is disruption of collagen fibres within the scar tissues at the dermal and sub-dermal level and breakdown of fibrotic bundles by needling. It stimulates fibroblasts for remodelling of extracellular matrix and formation of collagen fibers. ${ }^{11}$ During the procedure, saline is filled in extracellular spaces while blood clot is formed at the site of injection which results in elevation of scars. The release of various growth factors as a result of needling also helps in extracellular matrix and collagen formation. ${ }^{12}$

Two related trials have been reported so far. The study in Iran was carried out by Bagerani and Smoller, where 12 cases were enrolled in the study out of which 10 (83,3\%) were females and $2(16.7 \%)$ were males. Mean scores at first session were $6.7 \pm 1.5,7.2 \pm 2.02,7.0 \pm 1.8$, and $7.2 \pm 1.01$ for ice pick, boxcar, rolled scars and pores, respectively. At the fifth session, the scores were $10.0 \pm 0.9,10.9 \pm 1.01,10.4 \pm 1.3$, and $10.3 \pm 1.3$, respectively. ${ }^{12}$ In another study carried out in Lahore (Pakistan), 30 cases were enrolled with 21 (70\%) females and 9 (30\%) males. At the baseline, their scar severity, lesion counting, and final scores were $4.9 \pm 1.47$, $42.17 \pm 17.89$ and $225 \pm 136.42$ respectively while after the tenth session, scores were $2.87 \pm 1.43,22.3 \pm 17.18$ and 84.47 \pm 114.91 respectively. ${ }^{11}$ These studies signify efficacy of saline without any side effects. 
Treatment of atrophic scars are challenging with no definitive cure. There are various treatment modalities for atrophic scars which are categorised into energy and non-energy-based techniques. Energy-based methods include ablative and non-ablative lasers, fractional radiofrequency, intense pulsed light and plasma skin regeneration while non-energy-based methods are micro-needling, dermabrasion, subcision, augmentation by fillers and chemical peels. ${ }^{13}$ All these techniques have varying degrees of efficacy with their pros and cons. As compared to saline injection they are more expensive and require expertise, as these procedures can cause erythema, pigmentation, infection, acne, scarring and various other side effects specific to the procedure. The side effects of chemical peels include severe inflammatory reaction, loss of epidermal barrier resulting in water loss and superadded infections like reactivation of Herpes and candidiasis while systemic toxicity secondary to cutaneous absorption like arrhythmias, hepatotoxicity, nephrotoxicity and respiratory depression has also been reported. ${ }^{14}$ Close monitoring of patients is required due to these afore mentioned side effects. ${ }^{15}$ Side effects of microneedling, dermabrasion and subscision include erythema, oedema and pain. ${ }^{16}$ Saline injection works in a similar manner as microneedling, dermabrasion and subcision but without above mentioned side effects. ${ }^{17}$ Laser causes hyperpigmentation, infections, scarring and acneiform eruptions as its main side effects. ${ }^{18}$ Post-procedure haematoma and infections are adverse effects of tissue augmentation with collagen, hyaluronic acid and autologous fat. ${ }^{19}$

Therefore, the decision for treatment depends on physician's skills, cost of treatment, severity of lesions, expectations of patient, psychological and emotional impact on patient, efficacy and side effects of treatment. The ultimate result of all these modalities is just improvement in depth and reduction in numbers of scars as complete cure is not possible. Before selecting and starting any procedure, it is important to discuss with the patient about his expectations, chances of success and after-effects of therapy. ${ }^{20}$

As prevention is better than cure, patients' education is a cornerstone in the prevention of acne scars. It can be achieved by increasing public awareness of acne vulgaris and its sequel through proper education programmes. Counsel the patient to avoid picking and squeezing acne. Treatment of acne should be started promptly with correct regimen according to its severity to control inflammation at earliest possibility, as severe acne like nodulocystic acne leaves severe acne scars. ${ }^{21}$

In this study, three patients also had atrophic scars secondary to trauma in their childhood on their face, in addition to postacne atrophic scars. On the request of these patients, trauma induced scars were also filled with saline injection. The response of treatment in these scars was remarkable and comparable to post-acne atrophic scars. This additional use of saline injection requires further studies so that dysmorphic traumatic old scars can also be treated with this cost effective and easily accessible modality. It may provide a better quality of life for non-affording patients. In this study DLQI was calculated which showed significant improvement with treatment. The major significance of DLQI is that it helps in early recognition of presence of psychiatric distress in patients like social embarrassment with frustration and poor self-esteem. ${ }^{22}$ Post-traumatic scars on exposed parts of the body like face are equally distressing as post-acne scars and have similar influence on DLQI. ${ }^{23}$

The major limitation of study is an incomplete cure from scars. Post procedure follow-up is also required for long term durability of effects. Furthermore, studies are required for comparison of saline injection response with other treatment modalities.

\section{CONCLUSION}

The management of post-acne atrophic scars with saline injections is a safe and economical procedure. But further studies are required to improve the efficacy and durability of this procedure for achieving better results and validity versus other treatment options. Additionally, the response of saline injection to old post-traumatic scars also requires further studies to deal with such psycho-social and cosmetic disfigurement using this modality.

\section{ETHICAL APPROVAL:}

Legal and ethical approvals were obtained prior to initiation of the research work from institutional review board committee of Jinnah Postgraduate Medical Centre, Karachi.

\section{PATIENTS' CONSENT:}

Informed consent is obtained from patients to publish the data concerning their case.

\section{CONFLICT OF INTEREST:}

None to disclose.

\section{AUTHORS' CONTRIBUTION:}

SK: Contributed to the design research, ethical approval, patient consent, data collection, analysis of the results and writing of the manuscript.

RG: Involved in planning, supervised the work and provided critical feedback.

SK: Helped shape the research and manuscript.

\section{REFERENCES}

1. El-Taieb MA, Ibrahim HM, Hegazy EM, Ibrahim AK, Gamal AM, Nada EA. Fractional erbium-YAG laser and platelet-rich plasma as single or combined treatment for atrophic acne scars: A randomized clinical trial. Dermatol Ther (Heidelb) 2019; 9:707-17.

2. Kircik LH. Re-evaluating treatment targets in acne vulgaris: adapting to a new understanding of pathophysiology. J Drugs Dermatol 2014; 13:s57-60.

3. Killey J, Simons M, Kimble RM, Tyack Z. Effectiveness of interventions for optimising adherence to treatments for the prevention and management of scars: protocol for a systematic review and meta-analysis. BMJ Open 2019; 9: e023904.

4. Hazarika N, Archana M. The psychosocial impact of acne vulgaris. Indian J Dermatol 2016; 61:515-20.

5. Dréno B, Bissonnette R, Gagné-Henley A, Barankin B, Lynde C, Kerrouche $\mathrm{N}$, et al. Prevention and reduction of atrophic acne scars with adapalene $0.3 \%$ benzoyl peroxide $2.5 \%$ gel in subjects with moderate or severe facial acne: results of a 6 - 
month randomized, vehicle-controlled trial using intra-individual comparison. Am J Clin Dermatol 2018; 19:275-86.

6. Al-Dhalimi M, Jaber A. Treatment of atrophic facial acne scars with fractional Er: Yag laser. J Cosmet Laser Ther 2015; 17: 184-8.

7. Chernyshov PV. The evolution of quality of life assessment and use in dermatology. Dermatology 2019; 235:167-74.

8. Fabbrocini G, Annunziata MC, D'arco V, De Vita V, Lodi G, Mauriello MC, et al. Acne scars: pathogenesis, classification and treatment. Dermatol Res Pract 2010; 2010:893080.

9. Karppinen SM, Heljasvaara R, Gullberg D, Tasanen K, Pihlajaniemi T. Toward understanding scarless skin wound healing and pathological scarring. F1000Res 2019; 8:F1000.

10. Connolly D, Vu HL, Mariwalla K, Saedi N. Acne scarring - pathogenesis, evaluation, and treatment options. J Clin Aesthet Dermatol 2017; 10:12-23.

11. Asghar A, Tahir Z, Ghias A, Iftikhar U, Ahmad TJ. Efficacy and safety of intralesional normal saline in atrophic acne scars. Annals of King Edward Medical University 2019; 25:1-4.

12. Bagherani N, Smoller B. Introduction of a novel therapeutic option for atrophic acne scars: Saline injection therapy. Global Dermatology 2015; 2:225-7.

13. Kravvas G, Al-Niaimi F. A systematic review of treatments for acne scarring. Part 1: Non-energy-based techniques. Scars Burn Heal 2017; 3:2059513117695312.

14. Handog EB, Datuin MS, Singzon IA. Chemical peels for acne and acne scars in asians: Evidence based review. J Cutan Aesthet Surg 2012; 5:239-46.

15. Soleymani T, Lanoue J, Rahman Z. A practical approach to chemical peels: A review of fundamentals and step-by-step algorithmic protocol for treatment. J Clin Aesthet Dermatol 2018; 11:21-8.

16. Bhargava S, Kumar U, Varma K. Subcision and Microneedling as an Inexpensive and Safe Combination to Treat Atrophic Acne Scars in Dark Skin: A prospective study of 45 patients at a tertiary care center. J Clin Aesthet Dermatol 2019; 12:18-22.

17. Schmitt L, Marquardt $Y$, Amann P, Heise R, Huth L, Wagner-Schiffler $\mathrm{S}$, et al. Comprehensive molecular characterization of microneedling therapy in a human three-dimensional skin model. PloS One 2018; 13:e0204318.

18. Arsiwala SZ, Desai SR. Fractional carbon dioxide laser: Optimizing treatment outcomes for pigmented atrophic acne scars in skin of color. J Cutan Aesthet Surg 2019; 12:85-94.

19. Riyat H, Touil LL, Briggs M, Shokrollahi K. Autologous fat grafting for scars, healing and pain: A review. Scars Burn Heal 2017; 3:2059513117728200.

20. Fife D. Practical evaluation and management of atrophic acne scars: tips for the general dermatologist. J Clin Aesthet Dermatol 2011; 4:50-7.

21. Hay RA, Shalaby K, Zaher H, Hafez V, Chi CC, Dimitri S, et al. Interventions for acne scars. Cochrane Database Syst Rev 2016; 4:CD011946.

22. Chuah SY, Goh CL. The impact of post-acne scars on the quality of life among young adults in Singapore. J Cutan Aesthet Surg 2015; 8:153-8.

23. Bhooshan LS, Devi MG, Aniraj R, Binod P, Lekshmi M. Autologous emulsified fat injection for rejuvenation of scars: $A$ prospective observational study. Indian J Plast Surg 2018; 51:77-83. 